\title{
Fünfzehn Jahre „Tellux“: Audio-Visuelle (AV-) Medien-Arbeit für Kirche und Gesellschaft in der Praxis
}

\author{
von Otto Erich Kress
}

Im Frühjahr 1960 wurde die „Tellux" Film GmbH. gegründet. Der maßgebliche Anstoß ging von Bischof Dr. Carl Joseph Leiprecht in Rottenburg aus - und zwar auf Anregung des Katholischen Filmwerks e. V., das schon damals dem kirchlichen Film-Verleih- und -Vertriebsbereich einen Produktionspartner zugeordnet wissen wollte. Anläßlich der Begründung einer Organschaft mit der Berliner Film- und Fernseh-Produktionsgesellschaft „Provobis“ $\mathrm{mbH}$. im Jahre 1965 erfolgte die Verlegung der Gesellschaft von Rottenburg am Neckar nach München. Aufgrund eines Kooperations-Vertrages mit Studio Hamburg arbeiten die "Provobis" und "Tellux" Film seit 1971 auch in Hamburg. Das 15jährige Bestehen der "Tellux" und die 10jährige Organschaft mit der „Provobis" Film GmbH. in Berlin und Hamburg geben Anlaß zu einer kritischen Bestandsaufnahme und zu Fragen nach dem Entwurf für die Zukunft.

Seit am 19. November 1971 die katholische Wochenzeitung „Publik“ mit der roten Schlagzeile "Publik ist tot" herauskam, sind Fragen und Meinungsäußerungen zum Thema kirchlicher Publizistik nicht mehr verstummt. Es ist so, als habe erst dieser Kollaps wieder die Notwendigkeit und fragwürdig gewordene Wirklichkeit kirchlicher Offentlichkeit ins Bewußtsein zurückgerufen und die Aufmerksamkeit darauf gelenkt, daß das Wirken der Kirche ihrem Wesen nach öffentlich sein muß, daß Offentlichkeit für das Wirksamwerden der Kirche sozusagen eine zwingende Notwendigkeit ist. Vor dem Hintergrund dieser Feststellungen kann es deshalb für die kirchliche Arbeit und die religiöse Praxis nicht gleichgültig sein, daß mit dem Tod von "Publik“ der fatale Hinweis fiel, das katholische Milieu wolle nicht gestört sein, der Katholizismus strebe ins Getto. Demgegenüber hat Michael Schmolke sicher richtig beobachtet, daß die von Alois Schardt für "Publik" in Anspruch genommene Forum-Funktion sich in der Praxis nicht als durchführbar erwies: der "runde Tisch", den „Publik" darstellen wollte, war keineswegs immer ausreichend, noch galt er manchen als einladend. ${ }^{1}$

Inzwischen sind im katholischen Verlagswesen und auch in der Kirchenpresse Modelle für eine Mediendienstleistungsgesellschaft und für Gemeinschaftsredaktionen entwickelt worden. Diese Uberlegungen lassen darauf schließen, daß Abwehrkräfte gegenüber zweifellos vorhandenen Einigelungstendenzen mobilisiert werden, die sich sowohl in verlegerischer als auch publizistischer Hinsicht auswirken könnten. $\mathrm{Ob}$ diese Aktivitäten freilich ausreichen, den Austausch von Ideen und die Ausstrahlungs- und Überzeugungskraft der kirchlich orientierten Publizistik lebendiger zu

Dipl. pol. Otto Erich Kress ist einer der drei Geschäftsführer der Tellux-Film GmbH; mit seiner engagierten Interpretation der Tellux- und Provobis-Aktivitäten macht er auf das Arbeitsgebiet katholischer AV-Medien-Produktion in der BR Deutschland aufmerksam. 
machen und zu steigern, muß sich erst noch in der Praxis zeigen. Wie, so ist aber zu fragen, haben sich diese alarmierenden Entwicklungen für die Kirche in jenen Bereichen ausgewirkt, die abseits des unmittelbar umstrittenen publizistischen und journalitischen Terrains liegen? Gemeint sind jene Aktionsfelder der Audiovision, die in neueren Darlegungen der Kommunikationspraxis einen immer bedeutenderen Rang zugesprochen erhalten ? $^{2}$

Die Fragestellung zielt konkret auf die bisherige Tätigkeit und die zukünftigen Aufgaben und Möglichkeiten der "Tellux" und der mit ihr seit 1965 verbundenen "Provobis", die die beiden Produktionsgesellschaften in den drei deutschen Filmund Fernsehzentren München, Hamburg und Berlin wahrnehmen. Unsere Frage klammert damit den ganzen Bereich der kirchlichen Mitwirkung am öffentlichrechtlich verfaßten Fernsehsystem der ARD und des ZDF (Rundfunkfreiheit und Rundfunkkontrolle) zum weitaus größten Teil ebenso aus wie auch alle Fragen, die sich im Zusammenhang mit audio-visueller Praxis auf dem Gebiet der Medienpädagogik und Mediendidaktik ergeben. Zu dem Komplex der „Rundfunkfreiheit" und "Rundfunkkontrolle" hat sich im Laufe des letzten Jahres eine breite Diskussion entwickelt. Deren Fort- und Ausgang ist nicht nur für die Beantwortung der Frage nach Offentlichkeitsbezug oder Gettomentalität der Kirche von ausschlaggebender Bedeutung. Vielmehr muß sie auch, wie Karl Forster bemerkt hat, den institutionellen Formen unseres Systems öffentlicher Medien so zugute kommen, daß Rundfunk und Fernsehen unserer Gesellschaft soviel kommunikativen Dienst leisten können, wie diese Gesellschaft zu ihrer Integration und zur Pluralität ihrer Selbstdarstellung braucht. ${ }^{3}$ Die Fortentwicklung der medienpädagogischen und mediendidaktischen Ansätze und Erkenntnisse muß dabei im Einflußbereich der Kirche mit dem fortschreitenden Prozeß zunehmender audio-visueller Information und Kommunikation Schritt halten. Die Kritikfähigkeit der Gemeinden muß sich an den Angeboten der öffentlich-rechtlichen und privaten Bildungs- und Unterhaltungsindustrie orientieren. Dialogfähigkeit und Selektionsvermögen gegenüber einer zunehmenden Flut von Bildern müssen geübt werden.

Vor dem Hintergrund dieses aktuellen Befundes von Kirche und Offentlichkeit im Bereich der audio-visuellen Medien planen, produzieren und synchronisieren "Tellux ${ }^{\text {* }}$ und "Provobis" seit fünfzehn Jahren Filme und Fernsehsendungen. Ende 1974 gibt es annähernd 500 Filme und T'V-Produktionen, die von diesen beiden Produktionsgesellschaften hergestellt wurden und die jederzeit verfügbar sind. Zum Jahresende 1973 waren 394 Titel archiviert und katalogisiert und auf diese Weise allen Interessenten auf Abruf zugänglich. In den Beratungen über katholische Publizistik, die durch das Ende von "Publik" angestoßen wurden, findet dieser Bereich jedoch u. E. keine ausreichende Berücksichtigung. (Eine Ausnahme ist die Gründung der „AV-Kommission ${ }^{* 4}$.)

\section{II}

Die publizistische Arbeit der Kirche und ihre Entfaltung nach innen und außen war und ist für die Gründer und die im weiteren Verlauf der Firmenentwicklung hinzugekommenen Gesellschafter die entscheidende Triebfeder für den Aufbau der "Tellux “ Film GmbH. Ihre universal-kirchliche Perspektive kommt in drei wesentlichen Diensten zum Ausdruck: 1. der Förderung des Dialogs innerhalb der Kirche 
durch geeignete Zielgruppen-Filme, 2. der Unterstützung des Dialogs zwischen Kirche und Welt durch Filme und Fernsehsendungen, 3. der Aktualisierung der Glaubenswahrheiten durch bestmögliche Interpretationen im AV-Bereich.

Die Schwerpunkte dieser Arbeit entsprechen der diesbezüglichen Feststellung der päpstlichen Pastoralinstruktion Communio et Progressio: „Wer immer in der Kirche Verantwortung trägt, muß ständig bestrebt sein, durch die Medien umfassende und wahrheitsgemäße Informationen zu vermitteln, damit man ein zutreffendes Bild von der Kirche und ihrem Leben erhält. ${ }^{\text {" } 5}$

Was die "Tellux “ Film in den Jahren ihres Aufbaus von 1962 bis heute zu realisieren versuchte, entspricht ferner auch den Leitgedanken, die in den Grundsätzen kirchlichpublizistischer Arbeit von der maßgeblichen Synodenkommission dazu veröffentlicht wurden:

„Der Dienst der Kommunikationsmittel für das Leben der Kirche besteht darin, daß sie den Dialog innerhalb der Kirche sowie zwischen Kirche und Welt ermöglichen und fördern. Die Kommunikationsmittel leisten eine wirksame Hilfe, die Glaubenswahrheiten zu aktualisieren, daß sie in der heutigen kulturellen und gesellschaftlichen Situation verstanden und aufgenommen werden. Die Kirche hat das Recht und die Pflicht, durch die Kommunikationsmittel in einen Dialog mit der Welt einzutreten, ihre Lehre und ihr Wirken Menschen innerhalb und außerhalb der Kirche verständlich zu machen und ihre Deutung der Zeichen der Zeit vor dem Hintergrund der Heilsgeschichte darzustellen. Die Form der kirchlichen Verkündigung und der übrigen kirchlichen Darbietungen in den Kommunikationsmitteln muß daher immer mediengerecht sein. ${ }^{\text {« }}$

Die Gesellschafter der "Tellux" Film GmbH. haben ihre bisherige Arbeit und die Entwicklung und Planung ihrer zukünftigen Tätigkeit unter die Leitgedanken der Konzilsdokumente wie der Synodenaussagen gestellt. Sie gehen davon aus, daß es eine kirchliche Verpflichtung gibt, in der Sprache der Zeit und auch mit den Mitteln der modernen Medien den Dialog in der Kirche und mit der Welt zu führen und die christliche Botschaft zu verkünden. Dem Firmenzweck und dem Selbstverständnis der „Tellux“ Film GmbH. entspricht die Absicht, bei der Erfüllung dieser Aufgaben im Bereich der Audiovision (Film, Fernsehen, Tonbildschauen, Multimedia) einen Beitrag in kirchlicher Trägerschaft zu leisten.

Die Konzeption und Durchführung von Dialog, Information und Verkündigung der Kirche in diesem mit hohen organisatorischen und finanziellen Anstrengungen verbundenen Bereich (AV-Medien) bedarf einer weitgehenden Übereinstimmung in der geistigen Zielrichtung und der kreativen und wirtschaftlichen Verantwortung. Die "Tellux “ Film GmbH. löst deshalb die Aufgaben mediengerechter AV-Produktionen durch das Engagement und die Bereitstellung des technischen Personals nach fachlichen Kriterien und mit dem sachlichen Instrumentarium der Film- und Fernsehwirtschaft.

Zu den Gesellschaftern gehören heute fünf Bistümer, nämlich die beiden GründerDiözesen Rottenburg und München, und die inzwischen hinzugekommenen Bistümer Münster, Essen und Berlin mit 51,14\% der Gesellschafter-Anteile. Bei einem Gespräch der bischöflichen Mitglieder der Publizistischen Kommission der Deutschen Bischofskonferenz mit den Gesellschaftern der "Tellux" hat Weihbischof Walther Kampe, Limburg, im Frühsommer 1974 die Anregung gegeben, daß sich alle deut- 
schen Bistümer an der Tätigkeit der "Tellux" beteiligen sollten. Das Katholische Filmwerk, der Sebaldus Verlag Nürnberg (Gong) und das Winfried-Werk Augsburg (Weltbild), beide als kirchennahe Verlage, halten gemeinsam weitere $17,14 \%$ der Kapitalausstattung. Das restliche Firmenvermögen entfällt mit $31,72 \%$ auf Mitarbeiter (O. E. Kress und B. J. Grote) und filmwirtschaftliche Beteiligungen (Studio Hamburg). Mit dem Beitritt neuer Gesellschafter (Erweiterung der gesellschaftlichen Basis) vom 27.10.1971 wurde die Erhöhung des Stammkapitals auf jetzt DM 350.000,- beschlossen. Die Zielsetzung der „Tellux “ ist seit ihrer Gründung, unabhängig von den wirtschaftlichen Dispositionen, beibehalten worden: Gestaltung kirchlicher Programmbeiträge für deutsche und ausländische Fernsehanstalten und Durchführung von speziell kirchlich- und religiös-orientierten Filmproduktionen einschließlich ihrer thematischen Entwicklung standen im Vordergrund. Daneben konzentriert sich die "Provobis“ auf die Ausdehnung bzw. Erweiterung des Produktionsrahmens in Themenbereiche gesamtgesellschaftlichen Interesses über Theologie und Kirche hinaus. Hier entstehen seit Jahren Filme zu Fragen aus Politik und Zeitgeschichte, dem Arbeits-, Sozial- und Berufsleben und zu Problemen aus Kultur und Wirtschaft, die auch im weiteren Sinne dem Dienst der Kirche an der Welt entsprechen. Diese Zuordnung des „Provobis“-Produktionsprogramms auf die Film- und Fernsehplanungen der "Tellux “ ist eine den Eigenarten des Filmemachens gemäße Form, Probleme des Glaubens in einem für alle Weltprobleme aufgeschlossenen Horizont glaubwürdig und überzeugend darzustellen. Der geistigen Reichweite soll zeit- und sozial-orientiertes Interesse entsprechen. Vier Fachredaktionen für Dokumentar- und Fernsehspiele, für Dokumentation und religiöse Serien, für allgemeine kulturelle Features und für Wirtschaftsfilme versuchen, nach diesem Konzept zu arbeiten. In ständigem Austausch und Gespräch mit Mitarbeitern inund ausländischer Fernsehstationen und mit Autoren und Regisseuren des internationalen Films bemühen sie sich, zur Entwicklung und Realisierung neuer Projekte zu kommen. In diesem Zusammenhang seien drei Überlegungen zu den Anfängen christlichen Fernsehspiels „handmade by Tellux", wie sie Bernd J. Grote für unsere Produktionschronik 73 aufgeschrieben hat, im Kontext mit Bert Brechts „... um verkäuflich zu sein, muß die Kunst heute erst käuflich sein" wiederholt:

Erstens: das Professionelle, das kann man lernen; seit 1967 haben wir gelernt, Autoren kennengelernt, Regisseure, Darstellerinnen, mehr Darsteller, Ateliers, das know-how, wie man in der Branche redet (hinten im Hals), Küßchen auf die Wange, haben allerdings eine unverfälschte Neigung zum Amateurhaften nie ganz und gar verdrückt. Die Dokumentarspiele über den Mainzer Bischof Ketteler und den Münsteraner Kardinal Galen waren fast Erfolge.

Zweitens: die Umstände, wer könnte das besser schreiben als Dieter Stolte? „Dem verstärkt spürbaren künstlerischen und journalistischen Impetus zur Authentizität, dem unmittelbaren Dabeisein, dem Aufbruch aus den vier Wänden der Studios auf die Straßen und Plätze ... steht die betriebs-wirtschaftliche Notwendigkeit der rationellen Nutzung geschaffener Produktionsmittel (insbesondere auch der stationären wie elektronischen Studios und Filmateliers) gegenüber. Oder schlagwortartig formuliert, dem publizistischen Eros das industrielle Management. ${ }^{\alpha}$

Drittens: das Eigentliche, hier wage ich nur Positionen zu skizzieren. Die eine wie einst das Jesuitendrama religiös-ethische Aufgaben übernahm, so sollten wir Schillers Slogan vom Theater als moralischer Anstalt ungeniert auf das Fernsehen 
übertragen und beherzigen. Die andere - (noch einmal aus Brechts „Radiotheorie“) „durch immer fortgesetzte, nie aufhörende Vorschläge zur besseren Verwendung der (Fernseh-)Apparate im Interesse der Allgemeinheir haben wir die gesellschaftliche Basis dieser Apparate zu erschüttern, ihre Verwendung im Interesse der wenigen zu diskutieren“.

Diejenigen, die es ein- und abzuschätzen vermögen, werden bestätigen, wie schwer es ist, so vielen widersprüchlichen Anforderungen gerechtzuwerden und mit so vielen retardierenden Einwirkungen auf die Produktion von Filmen zurecht zu kommen. Die Fernsehspiel-Reihen "Patienten“, "Gleichnisse“, „Zwischenstationen" und jetzt "Rufzeichen“ (Frühjahr 1975) können daher auch nur Stationen auf dem Weg zu besseren, $d$. h. verständlichen und überzeugenden Chiffren von Verkündigung sein.

\section{III}

Bei der Beurteilung der bisherigen Firmentätigkeit muß berücksichtigt werden, daß die „Tellux" gemeinsam mit der "Provobis" im Bereich des AV-Sektors mit anderen Produktionsgesellschaften im Wettbewerb steht und daß ihre wirtschaftliche Basis von den Ergebnissen der Produktion abhängig ist. Die Auftraggeber von Filmen und Fernsehsendungen erwarten, daß die Filmherstellung von der Entwicklung des Drehbuches bis zur Ablieferung der einsatzfähigen Theater- bzw. Sendekopie zu den geringstmöglichen Kosten erfolgt. Andererseits hängt die gesicherte und gediegene Weiterentwicklung einer Film- und Fernsehproduktion davon ab, daß eine ausreichende finanzielle Grundlage vorhanden ist, die auch durch unvermeidbare oder schwer vorhersehbare Rück- und Fehlschläge nicht in Gefahr gebracht werden kann. Was allein die Aufnahme von Produktionen im Bereich der Entwicklungsländer oder einer internationalen Kooperation oder auch nur eines Spielfilmexperiments, wie etwa des "Francesco de Dio" von Wolfgang Suttner, in das Repertoire für die Bilanz einer eher kleinen bis mittleren Produktionsgesellschaft wie "Tellux “ bedeutet, kann hier nicht im einzelnen beschrieben werden. Erwähnung verdient allenfalls, daß die Gesellschafter der "Tellux “ seit Jahren umfangreiche Rückstellungen (Gewinnverzichte) durchführen, aus denen bisher die Kosten neuer Projekte etwa auf dem Gebiet der Kinderkatechese, der diözesanen Filmarbeit und des allgemeinen gesellschaftlichen Engagements der Kirche wenigstens teilweise bestritten werden konnten. Auch eher experimentelle Weiterentwicklungen im Bereich des Fernsehprogramms, wie beispielweise die Meditations-Sendungen des ZDF an Feiertagen, hätten von der "Tellux “ nicht aufgegriffen und betreut werden können, wenn ausschließlich auf die in diesem Falle nicht kostendeckenden Finanzmittel der Fernsehanstalt als Auftraggeber hätte zurückgegriffen werden müssen. Aus diesen Erfahrungen und Resultaten läßt sich nur der Schluß ziehen, daß die privatwirtschaftliche Struktur der Firmengruppe "Tellux-Provobis“ die einzige verläßliche Gewähr dafür bietet, daß die bisherige Produktionstätigkeit ohne Gefahr finanzieller Einbußen fortgesetzt werden kann. Eine wichtige, wenn nicht unerläßliche Voraussetzung dafür ist, daß die Produktionsarbeit der "Tellux" im Bereich von Film und Fernsehen auf ein wachsendes Verständnis einer nicht nur mäzenatisch-, sondern auch verkündigungs-orientierten Kirche rechnen kann. Nur unter dieser Voraussetzung vermag die "Tellux" Gewähr zu bieten, zu marktgerechten Preisen wichtige kirchliche Produktionsaufgaben (Schule, Jugendarbeit, Erwachsenenbildung, Sozialarbeit, Kindererziehung, Altenpflege, Gastarbeiterseelsorge, Priester- und Lehrerausbildung 
seien nur als dringlichste Einsatzgebiete und Anwendungsbereiche genannt) im AVBereich $\mathrm{zu}$ entwickeln, durchzuführen und mit kostenorientierten und aufwanddeckenden Ergebnissen abzuschließen. Die heute bei "Tellux " vorhandene Ausstattung an Kapital und Technik gewährleistet jedenfalls, daß die Entwicklung und Herstellung audio-visuellen Materials für Seelsorge, Katechese und Bildungsarbeit keine unüberwindlichen Probleme mehr aufwirft, wie und woher diese Mittel und das unerläßliche know-how zu beschaffen sind. Das Problem ist allenfalls, daß es in der Kirche zu wenige Auftraggeber gibt. Denn was Filme für den Bereich der Verkündigung vermögen und wie sie einzusetzen und auszuwerten sind, ist noch immer nicht genügend bekannt bzw. akzeptiert. ${ }^{7}$ In der Tat kann nicht deutlich und nachdrücklich genug darauf hingewiesen werden, daß die deutschen Fernsehanstalten Jahr um Jahr durch eine beachtliche Anzahl von Produktionen speziell aus dem Themenbereich der Welt des Glaubens zu den wichtigsten Auftraggebern der "Tellux" gehören. Und hier ist es wiederum vor allem das ZDF, dessen Produktionspolitik speziell auch im Bereich der kirchlichen Programmsparten auf Zusammenarbeit und ein ausgewogenes Verhältnis von Eigen- und Auftragsproduktionen hinausläuft.

Das ZDF hat diese Ausgewogenheit in den vergangenen Jahren durchzuhalten versucht, manchen politischen und finanziellen Anfechtungen zum Trotz. Die Programmverantwortlichen, vornehmlich der Intendant Prof. Holzamer, die Fernsehdirektoren Viehöver und Prager, haben in kontinuierlicher Folge erklärt, sie wollten und könnten nicht auf den von den freien Produzenten zu erstellenden Programmanteil verzichten. Man kann erwarten, daß sie sich zu einem solchen Verzicht, nicht einmal zu einer erheblichen Einschränkung der Auftragsvergabe an freie Produzenten, auch nicht durch die - ohnehin falschen - Hinweise bewegen lassen, die zunehmende Finanzierungs- und Finanznot der Anstalten, nicht zuletzt entstanden durch die sich zusammenballende Steuerbelastung, könne durch Eigenproduktionen gemindert werden. Eigenproduktionen der Anstalten sind, wie alle Sachkenner wissen, nicht billiger als Auftragsproduktionen (sie erscheinen nur, wenn man die Fixkosten der Anstalten nicht berücksichtigt, billiger). Die freien Produzenten können also in ihrer Gesamtheit damit rechnen, daß ihr Programmanteil in etwa bestehen bleibt, daß sie auch künftig eine angemessene Quote aus dem Programmvolumen erhalten werden. Die "Tellux" wird deshalb auch weiterhin davon ausgehen, daß sie innerhalb dieser Quote ihren Anteil erhalten und in Zukunft steigern kann, wenn sie in ihren Produktionen ihrem speziellen kirchlichen medien-politischen Auftrag nachkommt, und dies in einer Qualität, die das ZDF und die Seher verlangen. Dieser Qualitätsanspruch ist unabdingbar. Wenn die Grundsätze für eine Gesamtkonzeption der kirchlichen Publizistik ${ }^{8}$ darauf hinweisen, daß die Kirche im Bereich der Medien in besonderem Maße auf das Engagement sachlich kompetenter und hinreichend auf die speziellen Aufgaben vorbereiteter Christen angewiesen ist, so ist dies nichts anderes, als die nachdrückliche Betonung des Qualitätsanspruches. Kein Verantwortlicher des heutigen Fernsehens, weder ein Programmdirektor noch ein Kirchenfunkredakteur wird heute wegen des kirchlichen Inhalts einer angebotenen Produktion auf seinen Qualitätsmaßstab verzichten: es gibt keine "gut gemeinte“ Produktion

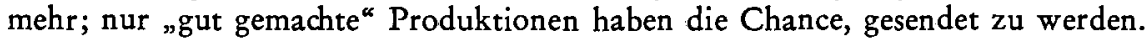

Man darf davon ausgehen, daß die Produktionen der "Tellux" - wie Branchenkenner und insbesondere auch Konkurrenten einräumen - zu diesen "gut gemach- 
ten " Produktionen gehören. Dies beweist schlüssig der Umstand, daß die Produktionen dieser kirchlichen Produktionsgesellschaft kontinuierlich immer wieder ins Programm aufgenommen werden.

Dabei ist von besonderer Bedeutung, daß die "Tellux-Provobis" nicht nur im Rahmen der von der Kirche mit verantworteten Sendungen ihren Platz gefunden haben und halten, sondern daß sie auch in anderen Programmbereichen (insbesondere den ZDF-Abteilungen Kultur, Jugend, Fernsehspiel, Dokumentarspiel, Chefredaktion) tätig sind.

Im Bereich der ARD liegen für die Präsenz kirchlich-bestimmter Produktionen auf längere Sicht Chancen für eine Verbreiterung und Vertiefung. Zum WDR (Köln), zum Südfunk (Stuttgart), zum Bayerischen Rundfunk (München) und dem Südwestfunk (Baden-Baden) bestehen intakte Arbeitsverbindungen. Die Rechtsprechung des Bundesverfassungsgerichtes hat darüber hinaus für diesen öffentlich-rechtlichen Sendebereich den Grundsatz entwickelt, daß die gesellschaftlich-relevanten Kräfte, und zu denen gehören die Kirchen, auch im Programm ihren Platz finden müssen. Damit die ARD dem Anspruch auf Ausstrahlung kirchlicher Sendungen gerecht werden kann, müssen die ARD-Anstalten mit einem vermehrten Programmangebot aus dem kirchlichen Raum bedient werden. Dies sollte langfristig zu einer verstärkten Annahme kirchlicher Programm-Angebote durch ARD-Anstalten führen, zumal die Struktur der ARD keineswegs der Annahme von Auftragsproduktionen, der Sendung von Produktionen freier Produzenten, entgegensteht. Allerdings wird dies nur gelingen, wenn der Weg hierzu über den bereits vorhandenen Einfluß auf kirchliche Sendungen gesucht wird, die Kirche selbst bereit ist, ihre Einflußmöglichkeiten auch tatsächlich zu nutzen und sie systematisch einzusetzen. Dabei könnte der "Tellux" die Aufgabe zufallen, diese Aktivitäten zu koordinieren."

Eine nicht unwichtige Rolle für die weitere redaktionelle und produktionstechnische Entwicklung der "Tellux" spielen aber neben den Fernsehanstalten auch die neuen angekündigten Medien des AV-Bereiches, die Bildplatte, die Bildtonkassette und das Kabelfernsehen.

Die Bildtonplatte und die Bildtonkassette sind zur Zeit technisch bei den Wiedergabegeräten noch nicht so ausgereift, wie vor einigen Jahren erwartet wurde. Sind aber die technischen Mängel behoben, so öffnet sich hier ein großer und sehr aufnahmefähiger Markt. Dieser darf und kann nicht der reinen Unterhaltung überlassen bleiben. Für ihn sollten kirchlicherseits rechtzeitig Programme entwickelt und bereitgestellt werden. Denn Bildplatte und -kassette sind, langfristig gesehen, interessante Medien. Gutachtliche Prognosen angesehener Wirtschaftsinstitute sind zwar widersprüchlich, lassen aber keinen Zweifel, daß es hier zu einem publizistisch und wirtschaftlich wichtigen Bereich öffentlicher Kommunikation kommen wird.

Einen noch größeren Platz als Bildplatte und -kassette kann in absehbarer Zeit das Kabelfernsehen einnehmen. Die Kommission für technische Kommunikation, welche der Bundespostminister ins Leben gerufen hat, erkannte bereits in ihren ersten Sitzungen, daß ungeachtet der außerordentlichen Kosten der Einführung des Kabelfernsehens (also der Verkabelung) die technische Entwidklung und die politische Meinungsbildung das Kabelfernsehen zur Realität machen wird. Dies bedeutet dann ein außerordentlich vergrößertes Spektrum des Informations- und des Unterhaltungsangebotes. 
Vom Kabelfernsehen wird ein wesentlich engerer Kontakt zwischen Seher und Sender erwartet als dies bei den großen öffentlich-rechtlichen Anstalten der Fall ist und sein kann. Gerade kirchliche Programme werden hier frühzeitig Modelle entwickeln müssen, die auf diesen Kontakt abstellen.

Zusammenfassend kann gesagt werden, daß die Produktion von Filmen und Fernsehprogrammen, die Beobachtung neuer Auswertungsmöglichkeiten im Medienbereich und die Erfüllung der dringlichsten Bedürfnisse aus den innerkirchlichen Bildungsund Sozial-Einrichtungen (die jetzt von der AV-Kommission des Verbandes der Diözesen nebenberuflich und im Ehrenamt gesammelt und kanalisiert werden) bereits ein immenser Aufgabenkatalog ist. Voraussetzung für ein erfolgversprechendes Medienkonzept ist aber, daß der kirchliche Mitwirkungsanspruch sich im Film und Fernsehen auch in unmittelbarer direkter Gestaltung äußert und auszudrücken vermag.

\section{IV}

In Anbetracht des Umfangs und der Wichtigkeit der Aufgaben, die von der Kirche im Bereich der AV-Medien angepackt werden müssen, stellt sich naturgemäß die Frage nach den bestmöglichen Wegen, die zu ihrer Bewältigung führen. Es ist erstaunlich, daß zu Fragen der Film- und Fernsehproduktion in unmittelbarer kirchlicher Verantwortung bisher keine Stellungnahme vorliegt. Was ist nun eine „kirchliche" Produktionsgesellschaft? Bedeutet die Tatsache, daß 68,28\% des Gesellschaftskapitals, also immerhin mehr als zwei Drittel des Firmenvermögens, sich in den Händen kirchlicher Gesellschafter befindet, daß die "Tellux " eine "kirchliche" Produktionsgesellschaft ist? Muß nicht die Frage gestellt werden, ob die "Tellux" nicht ein Pflänzchen jener Epoche ist, in der noch eigene Zeitungen, eigene Radiostationen, überhaupt eigene katholische Institutionen aller Art erstrebt wurden, und daß damit dem später so kritisierten Dualismus Vorschub geleistet wurde? Mit der Antwort auf diese Frage haben es sich die Gesellschafter und Mitarbeiter der "Tellux " nicht leicht gemacht. Sie fällt dennoch zu Gunsten einer kirchlichen Trägerschaft aus, weil die Bejahung der kirchlichen Mitverantwortung eine Konsequenz aus der täglichen film- und fernsehpublizistischen Praxis ist.

Das Engagement für die Entwicklung von Themen und Stoffen in Bezug auf das wesensgemäß Christliche des redaktionellen Gesamtkonzepts ist schließlich unteilbar. Mit anderen Worten, der Offenheit etwa für Probleme der Berufsberatung, Rentenversicherung, Sozialpädagogik, des Gemeinschaftskunde-Unterrichts, der Verbraucherberatung oděr des Denkmalschutzes bedarf es im gleichen Maße, wenn Film-Layouts über eine Indianergemeinde am Amazonas oder ein Katechetik-Modell zur "Sache Jesu" recherchiert werden müssen. Es ist deshalb nur folgerichtig, wenn aus diesem Befund auch Schlußfolgerungen für den gesellschaftlichen, den rechtlichen und wirtschaftlichen Status der Produktion gezogen werden. Hier dürfen es die unmittelbar Beteiligten nicht an gutem Willen und nicht an Konsequenz fehlen lassen. Für die Gesamtkirche ist dabei die Entscheidung nicht schwieriger als für die bisherigen Gesellschafter und Träger der "Tellux “, denn die Kirche muß auf die Entwidklung der AV-Medien als eine dem Wesen der Verkündigung assistierende Einrichtung auch direkten Einfluß nehmen können. Dies gilt insbesondere auch - neben sicher 
vielen Aufgaben, die um Bedeutendes wichtiger sind - für die Vertretung kirchlicher Standpunkte nach außen (sic!) bis beispielsweise hin zur Wahrnehmung des Rechts auf Gegendarstellung. Eine solche Inanspruchnahme des Mediums Film kann doch gegebenenfalls einmal nur dann möglich sein, wenn die Kirche sowohl technisch als auch wirtschaftlich einen eigenen Zugang zur Organisation und Abwicklung der Produktion hat.

Die Entscheidung für eine "kirchliche“ Produktion sollte und darf jedoch nicht verwechselt werden mit einer Option für eine „Kirche nach innen“, als bedeute dies, Audiovision für den kirchlichen Hausgebrauch zu konzipieren. Das Gegenteil, die Offenheit nach innen und außen muß das Ziel sein. Auch bei kritischer Zurückhaltung gegenüber einem kirchlichen Produktionsengagement sollten die Argumente für die konkreten Möglichkeiten der "Tellux" nicht gering geschätzt werden. Das Sprechen von Gott in der heutigen Gesellschaft kann die Kirche auch im AV-Bereich nicht allein mittelbar besorgen lassen, indem sie sich um einen guten Kontakt mit den Sendern bemüht, Autoren und Regisseure in die Fernseh-Anstalten schickt oder an Fernsehstudios und Film-Ateliers vermittelt - und was noch alles sonst an wichtiger Konsultation und Kooperation möglich ist. Damit keine Mißverständnisse enstehen: alle diese aufgezeigten Wege praktischer Zusammenarbeit sind verdienstvoll und müssen weiter beschritten und nach Möglichkeit breiter, selbstverständlicher, flüssiger werden, aber sie bedürfen der Ergänzung durch eigene beifallheischende Leistungen auch und vor allem auf dem Gebiet einer selbstverantworteten und überzeugend präsentierten Produktion. Niemandem sollte der Aufwand hierfür zu schade sein. Schon um der Glaubwürdigkeit willen sollte auch das Infragestellen eines respektablen Einsatzes von Mitteln auf dem Gebiet der AV-Publizistik endlich ein Ende haben. Wer aus den Sitzungen und Haushaltsberatungen der Sendeanstalten weiß, mit wie hohem Aufwand Programme gemacht werden müssen, wer dort streitet, daß das Budget für kirchliche Sendungen nicht zu klein gerät, der müßte den Mut haben, beim eigenen Bedarf, da wo Kirche mit eigenen Mitteln antreten muß, nicht alles dem Rotstift zum Opfer fallen zu lassen. Mit FeigenblattAktionen ist es nicht mehr getan, wenn nicht durch Unschlüssigkeit und falsche Sparsamkeit schwerer Schaden entstehen soll.

Um der Welt willen darf sich die Kirche mit ihrer Botschaft im Dialog mit der Gesellschaft nicht an den Rand der Offentlichkeit drängen lassen. Die Quintessenz des „aggiornamento“ heißt, mit den Menschen von heute in der Sprache ihrer Zeit im Gespräch zu bleiben. Die Kirche muß mit dem Film und dem Fernsehen leben, sie muß alle Medien der Audiovision in ihre praktische Arbeit einbeziehen. Das gilt - auch - für den Produktionsbereich, weil von der Verfügbarkeit möglichst verschiedener Themen und Titel der Einsatz von Filmkopien etc. in den Gemeinden, Verbänden und Schulen abhängt. Unbeschadet der Möglichkeiten, die sich der Kirche in den Massenmedien durch die Heranbildung geeigneter Mitarbeiter und deren Mitwirken in den Funkhäusern und an sonstigen Produktionsstätten bieten, sollten der Ausbau und die Weiterentwicklung einer eigenen leistungsfähigen Produktion weiter betrieben werden, die sich der kreativen, wirtschaftlichen und filmtechnischen Möglichkeiten des AV-Bereiches mit einem jeweils angemessenen Aufwand anzupassen vermag. Unter Ausbau und Weiterentwicklung ist zu verstehen, daß kirchlicherseits die Entwicklung und Herstellung brauchbarer AV-Medien mehr als bisher gefördert wird. Dies muß vor allem durch die Entwicklung und Durchführung einer großen 
Zahl von Filmen für den Gebrauch in den Gemeinden geschehen. Die Beteiligung an Kooperationsprojekten mit den Rundfunkanstalten und anderen öffentlichen Einrichtungen des Bildungswesens muß zielbewußt in Angriff genommen und ausgebaut werden. Hierher gehören vor allem erst einmal Filme für die Elternbildung, die Jugendarbeit und die sozialen Dienste der Kirche. Die Konzentrierung aller kirchlichen Produktionsaufgaben auf einen vorhandenen und in kirchlicher Trägerschaft aufgebauten Produktionsapparat sollte nicht länger umstritten sein.

Je schneller sich die unmittelbar an der kirchlichen Medienpolitik Beteiligten auf ein konkretes Konzept einigen, desto schneller wird man sich in der Kirche darüber klar werden, welche Möglichkeiten und Vorteile sich bieten, wenn kirchlicherseits die Kräfte gebündelt und die vorhandenen Mittel planmäßig und gezielt eingesetzt werden. Dabei geht es schlicht und einfach um Prämissen, die für den Erfolg kirchlicher Medienpraxis von ausschlaggebender Bedeutung sind. Der Einsatz kirchlicher Mittel muß über eine eigene Produktionsgesellschaft erfolgen, um die finanziellen Mittel so lange wie möglich im eigenen Bereich zu halten und damit die Potenz eigener Einrichtungen im Gesamtgeflecht der Fernsehanstalten und des Medienverbundes zu stärken. Damit wird kirchliche Wirksamkeit in der Gesellschaft deutlicher zur Geltung gebracht. Dadurch wird aber kostengünstige, marktgerechte und konkurrenzfähige Produktion auch in der Zukunft möglich; die wirtschaftlichen Mittel wiederum können zur Stärkung der kirchlichen Produktionskapazität und des ideellen Einflusses in den aufgezeigten Medienbereichen herangezogen werden.

Der Auf- und Ausbau einer bedürfnisgerecht zu handhabenden Operationsbasis für gezielte Maßnahmen und deren Ausnutzung im Medienverbund läßt sich auf diese Weise zielstrebig weiter verfolgen. Von allen diesen Überlegungen und Erwägungen ist - wohlgemerkt - in den bisher zugänglichen „kirchlichen“ Medienpapieren nach dem Tod von "Publik" wenig zu spüren gewesen - Film, Fernsehen und sonstige AV-Medien werden eher prophylaktisch in Randbemerkungen zitiert, soweit es sich überhaupt um Konzepte handelt, die eigene Aktivität voraussetzen oder zum Ziel haben. Eine kirchliche Produktionstätigkeit in diesem Bereich darf auf diese Weise aber nicht in die Bezirke eines Milieus oder Gettos verdrängt werden, auch nicht von jenen theologischen Medien-Theoretikern, die meinen, „die Sache Gottes werde nicht durch Ơffentlichkeitsbewußtsein und Aktivität der Kirche öffentlich, weil die Sache Gottes (aus sich heraus ehe schon) öffentlich sei. ${ }^{\text {" }}$ "So sehr der Denkansatz Aufmerksamkeit verdient, daß der Aufbau kirchlicher Strukturen im Medienbereich nicht zu "Hektik und Angstlichkeit unkritischen und atemlosen Kommunikationswillens "11 verführen darf, so sehr bedarf es der Unbefangenheit und Offenheit für eine differenzierte Ausgestaltung aller Kontaktmöglichkeiten mit den Medien. $\mathrm{Zu}$ diesen Kontaktmöglichkeiten gehört die entschlossene Fortsetzung aller Bemühungen, die gestalterischen Möglichkeiten im Bereich des Films und des Fernsehens zu nutzen und beherrschen $\mathrm{zu}$ lernen, in journalistisch-publizistischer, künstlerischer, technischer und wirtschaftlicher Hinsicht. Auf diese Weise läßt sich jedenfalls das von Giselbert Deussen beschriebene Problem einer „Distanz ohne Distanzierung und einer Nähe ohne Anbiederung "12 katholischer Publizistik noch am ehesten lösen. Es geht unverändert darum, daß es in den "Grenzbereichen " der audiovisuellen Produktion nicht zu einem Kommunikationsbruch zwischen Kirche und Gesellschaft kommt: der Abmarsch ins Getto muß ebenso verhindert werden, wie das rat- und tatlose Schweigen einer sich unverstanden fühlenden Kirche im Milieu. 
1. Michael Schmolke: „Publiks“ Ende: Schwächen unserer pluralistischen Kommunikationsverfassung. In: Ders. (Hrsg.), Publik - Episode oder Lehrstück?, Paderborn 1974, S. $12-20$, hier S. $16 \mathrm{ff}$.

2. Bernd Schiphorst, Audiovision - Portrait einer Branche, Hamburg 1974, S. 7 ff.

3. Karl Forster, Der Rundfunk und die gesellschaftlich relevanten Gruppen. In: „FunkKorrespondenz" Nr. 41 v. 9. 10.1974 (Beilage), S. 3.

4. Die "AV-Kommission" war 1972-1974 mehrfach Gegenstand der Beratungen der Publizistischen Kommission der Deutschen Bischofskonferenz. (D. Red.)

5. Communio et Progressio, Nr. 123, in: Franz-Josef Eilers, Karl Höller, Josef Hosse, Michael Schmolke (Hrsg.): Kirche und Publizistik, Dreizehn Kommentare zur Pastoralinstruktion „Communio et Progressio“. Mit dem deutschen Originaltext.

Mit einem Vorwort von Andreas Maria Deskur, Sekretär der Päpstlichen Kommissions für die Instrumente der Sozialen Kommunikation (Beiheft $1 \mathrm{zu}$ „Communicatio Socialis"), Paderborn 1972, 199 Seiten, hier S. 179.

6. Vgl. „Synode“, Komm. Ber. VI, Grunds. 3.5, 3.5.1 und 3.6 von 8-71-27 ff.

7. Auf diesen Sachverhalt hat Wilhelm Schätzler in anderem Zusammenhang hingewiesen, wobei die spezifischen Bewußtseinsschranken der herkömmlichen Theologie besonders deutlich hervortreten. In: „Film-Dienst“ Jg. 27, Nr. 8.

8. Vgl. „Synode“, Komm. Ber. VI, Grunds. 3.2 von 8-71-27 ff.

9. Diese Uberlegungen stammen von RA Dr. Reinhold Kreile, MdB und Syndikus des Fernsehproduzentenverbandes (Gutachten über die Stellung der "Tellux-Provobis“ im derzeitigen und künftigen Medienbereich, Privat-Druck 1974).

10. Traugott Stählin, Offentlichkeitsarbeit der Kirche und Massenkommunikation, in "Communicatio Socialis", 7:1974, S. 108.

11. Ders. a.a.O.

12. Giselbert Deussen, Kirche zwischen Offentlichkeit und Getto: soziologische und publizistische Perspektiven. In: „Communicatio Socialis“, 7:1974, S. 133.

\section{S U M M A R Y}

After fifteen years of existence of "Tellux “ Film Production (Munich and Hamburg), and after ten years of cooperation with "Provobis“ Films (Berlin), the Director of the companies tries to review it critically, and puts questions for the future. Both companies have about 500 available films and TV productions towards the end of 1974 . This work has been done in relation with the Church, but until now it has never been accepted into a scientifically worked out and reflected concept for communications work of the German Church. The author asks for a further development and streamlineing of all ecclesiastical productions via the already existing means.

This would help in the pastoral work in the parishes. He sees the basic stand between communications and Church together with Giselbert Deussen in "Distance without distance, and nearness without familiarity".

\section{RESUMEN}

El autor analiza críticamente la actuación de la «Tellux» Film, Sociedad de responsabilidad Limitada (Munich/Hamburgo), con motivo del décimo quinto aniversario de su creación y del décimo de su cooperación con la sociedad cinematográfica "Provobis» (Berlin/Hamburgo), y se pregunta por un modelo para el futuro. A pesar de que hasta finales de 1974 ambas productoras sacaron al mercado unas 500 películas para cine y televisión, constata que esa 
producción, realizada en el ámbito de la influencia eclesiástica, no encontró hasta ahora un lugar específico en el concepto científico y empírico de la estrategia publicística eclesiástica oficial. El autor es partidario de la realización y desarrollo, así como de la concentración de todas las producciones eclesiásticas de este tipo en el marco de un sistema de producción ya existente y vinculado a la jerarquía eclesiástica, sobre todo con vistas al aprovechamiento de la práctica comunitaria. Junto con Giselbert Deussen describe la actitud básica entre los sectores publicistas y la Iglesia como «distancia sin distanciamiento y aproximación sin intimidad».

\section{RESUME}

L'auteur entreprend, à l'occasion de $15 \mathrm{e}$ anniversaire de sa fondation, un inventaire critique de la "Tellux" Film GmbH. (Munich/Hambourg) et de ses dix années de coopération avec la «Provobis» Film GmbH. (Berlin/Hambourg); il pose des questions au sujet des projets d'avenir. Bien que les deux sociétés de production disposent, fin 1974, de près de 500 films et productiones télévisées, il constate que ce rendement filmé et télévisé, pour autant qu'atteint dans l'étroit domaine d'influence des représentants de l'Eglise, n'a jusqu'à présent pas encore trouvé de place dans le concept scientifique et empirique de la stratégie ecclésiastique officielle en matière de publicistique. L'auteur plaide pour l'achèvement et l'élargissement, de même que la concentration de toutes les tâches de productions ecclésiastiques de ce domaine dans un appareil de production construit, déjà existant, et sous la responsabilité de l'Eglise - surtout aussi à l'usage de la pratique collective. Avec Giselbert Deussen, il voit l'attitude fondamentale entre le domaine publicistique des mass-media et l'Eglise dans une «distance sans se distancer, et dans un rapprochement sans se faire des anvances». 